\title{
Una aproximación a los roles de las mujeres en sus lugares de reinserción durante la pandemia por la COVID-19
}

\author{
Silvana Duharte Barreda \\ Estudiante de Antropología en la Pontificia Universidad Católica del Perú (PUCP). \\ e-mail: sduharte@pucp.edu.pe
}

\section{Resumen}

La crisis sanitaria y la pandemia generadas por la COVID-19 han tenido diversas consecuencias entre peruanos y peruanas. Durante los meses de abril y mayo, miles de migrantes que vivían en Lima y otras ciudades, donde residían por razones laborales o de estudios, retornaron a sus lugares de origen con el objetivo de encontrar mayor estabilidad y tener sustento familiar. En el proceso de reinserción a estos espacios, las mujeres, específicamente, han visto transformadas sus responsabilidades domésticas y laborales. El objetivo principal del presente trabajo es describir y explicar los roles $\mathrm{y}$ acciones que las mujeres han adoptado en los lugares a los que retornaron, ya que es necesario poder tener primeros acercamientos sobre las rutinas y experiencias que la población femenina migrante está experimentado y experimentó durante los primeros meses del 2020. La metodología utilizada fue de corte etnográfico virtual y se utilizaron entrevistas semiestructuradas para poder cumplir con los objetivos de investigación. Tomando en cuenta las experiencias que las informantes que participaron en la investigación compartieron sobre su proceso de retorno, es posible tener una aproximación a los cambios que han experimentado las mujeres durante los primeros meses de pandemia. Este artículo proporciona algunos primeros resultados y propone continuar la investigación en torno a la temática para poder comprender de manera más compleja la realidad de las mujeres retornantes y la transformación de sus roles.

\section{Palabras clave}

Pandemia, retorno, mujeres, roles, deberes.

Nota editorial: el presente artículo fue recibido el 17-01-21 y aprobado el 08-02-21.

https://doi.org/10.18800/anthropia.2021.005 


\title{
An approximation to women's roles in their places of reinsertion during the COVID-19 pandemic
}

\author{
Silvana Duharte Barreda \\ Anthropology student at the Pontifical Catholic University of Peru (PUCP). \\ e-mail: sduharte@pucp.edu.pe
}

\begin{abstract}
The health crisis and the pandemic generated by COVID-19 has had various consequences among peruvians. During the months of April and May 2020, a large number of people decided to return to their places of origin with the objective of finding stability and family support. In the process of reintegration into these spaces, women, specifically, have seen their domestic and work responsibilities transformed. The main objective of this article is to describe and explain the roles and actions that women have adopted in the places to which they returned, since it is necessary to be able to have a first approach to the routines and experiences that the migrant female population is experiencing during the pandemic and during the first months of 2020. The methodology used based on a virtual ethnographic approach and semistructured interviews were used to be able to fulfill the research objectives. Taking into account the experiences that the informants who participated in the research shared about their return process, it is possible to have an approximation to the changes that women have experienced during the first months of the pandemic. This article presents some first results and encourages continuing the research around the subject in order to understand the reality of women returnees and the transformation of their roles in a more complex way.
\end{abstract}

\section{Keywords}

Pandemic, return, women, roles, chores. 


\section{Introducción}

En el Perú, la pandemia de la COVID-19 ha demostrado la incapacidad del Estado y sus servicios para enfrentar crisis exógenas, además de evidenciar las brechas y desigualdades sociales que existen entre los peruanos y peruanas. Debido a las altas probabilidades de contagio, ineficiencia de los servicios de salud y una deficiente aplicación de políticas públicas, la población tuvo que adaptarse a ciertos lineamientos restrictivos establecidos por el Estado. En general, las nuevas condiciones sociales, laborales y económicas productos de la COVID-19 tuvieron un impacto importante en la manera en la que las personas organizaban su vida. Durante los meses de abril y mayo, en el país se empezaron a llevar a cabo movilizaciones de miles de personas que decidieron dejar los lugares a los que habían migrado por razones laborales o de estudios, y retornar a sus lugares de origen donde se encontraban sus familias y, probablemente, donde podrían encontrar mejores condiciones de vida durante los meses de cuarentena.

Desde una perspectiva antropológica, el tema de los retornantes permite analizar y revisar las ideas en torno a las movilizaciones del campo a la ciudad y las actividades económicas que realizan los individuos en los lugares a los que llegan. Las migraciones son comunes en la realidad nacional y han estado presentes a lo largo de la historia del país. En la actualidad, las personas en zonas rurales no solo se dedican a actividades agrícolas, sino que participan de diferentes esferas económicas fuera de sus lugares de origen durante las épocas en las que no hay cosechas. Esto genera que haya nuevas dinámicas sociales, asentamientos y estrategias económicas. Lo relevante es que el contexto de pandemia resaltó estas movilizaciones y sus implicancias, por lo que es necesario que se describan y analicen las trayectorias y estrategias que utilizaron los individuos, así como también reflexionar en torno a las posibles decisiones futuras que tomarán y la forma en la que se insertaron nuevamente en sus contextos locales.

En esta línea, mi interés es centrarme en un tema transversal a estas movilizaciones: la experiencia de las mujeres con respecto a este fenómeno. Las estrategias y la forma de reinserción en el lugar de retorno son distintas para hombres y mujeres debido a las responsabilidades y expectativas que se tiene sobre ellos y ellas. Las experiencias cambian y se viven de maneras diversas, las cuales son necesarias de ser estudiadas. Generalmente, se asocia el papel de la mujer en ámbitos rurales a uno doméstico; sin embargo, en la actualidad, estas se dedican a diversas actividades económicas, así como también pueden movilizarse a otros lugares por motivos académicos. Es necesario que las estrategias en torno a la migración y la experiencia por la que pasaron las mujeres durante los retornos y su reinserción por la pandemia sean estudiadas, ya que, probablemente, estas estén unidas a otros temas como, por ejemplo, las expectativas de género, las responsabilidades y roles que deben cumplir en el hogar u otros espacios. En ese sentido, mi interés se centra en describir, y explicar los roles y acciones que las mujeres han adoptado en los lugares a los que retornaron. 
Para poder tener un mejor acercamiento a los roles que las mujeres desempeñaron y desempeñan en sus hogares de retorno, fue necesario plantear tres objetivos específicos que permitirán entender la problemática de una manera más completa: en primer lugar, identificar las acciones y tareas que realizan las mujeres en los lugares de retorno desde que regresaron de Lima; en segundo lugar, identificar las tensiones que perciben las mujeres sobre sus roles en los lugares en los que se reinsertaron; por último, explorar las percepciones que tienen las mujeres migrantes sobre las actividades y roles que desempeñan en sus lugares de origen desde el retorno.

\section{Metodología y acercamientos teóricos}

La metodología utilizada para poder desarrollar el trabajo fue de corte etnográfico virtual. La comunicación con las informantes se pudo lograr a través de llamadas telefónicas y videollamadas vía WhatsApp. Con respecto a los sujetos de estudio, se planteó que fueran mujeres de entre 17 a 40 años que migraron o vivían en Lima y que, debido a la pandemia, decidieron regresar a sus lugares de origen, y reinsertarse en las actividades económicas y sociales en tales espacios. Durante el periodo del trabajo de campo, se mantuvo contacto con tres informantes. En primer lugar, se entrevistó a Rosa Chávez, madre de familia y trabajadora que se movilizó de Lima a la ciudad Andahuaylas con su esposo y su hijo. La segunda informante fue Fiorella Mendoza, quien retornó a Lunahuaná con su familia durante unos meses. Finalmente, la tercera entrevistada que formó parte de la investigación fue María Valdez, madre de familia que regresó a Yapa en Cajamarca. Es necesario resaltar que, en el presente trabajo de investigación, se les pidió a las informantes su consentimiento para ser grabadas durante las entrevistas. Asimismo, también se preguntó si estaban de acuerdo con que la información que brinden sea utilizada para los propósitos de esta investigación y, por motivos de protección de su identidad, se han utilizado seudónimos. Por otro lado, para comprender mejor las vivencias y roles que experimentan las mujeres retornantes, es necesario explicar brevemente algunas ideas y conceptos teóricos sobre los cuales se ha reflexionado en torno a las vivencias de las mujeres y su situación a raíz de la pandemia.

En primer lugar, Paerregaard (2007) centra su estudio en las migraciones transnacionales a España y Argentina de mujeres peruanas. Tiene como objetivo explicar las formas en las que se integran a los nuevos países a través del trabajo y las estrategias que utilizan para poder adaptarse al nuevo ambiente. Una de sus conclusiones principales y más relevantes es que la mayoría de las mujeres migran con expectativas de poder mantener y enviar dinero a su familia para que puedan vivir cómodamente en Perú. Además, se espera que, en algún momento y en determinados casos, puedan iniciar nuevas vidas en estos lugares. Es importante tener una visión y comprensión de las investigaciones sobre las migraciones transnacionales de mujeres peruanas, puesto que comparten diversos aspectos y dimensiones que se identifican y analizan también en la literatura que se centra en las movilizaciones dentro del país por parte de esta población. Como mencionan diversos autores, las 
migraciones que se realizan del campo a la ciudad generalmente se deben a motivos académicos y a la búsqueda de trabajo. La experiencia de las mujeres con respecto a la inserción laboral en diversas ciudades, incluida Lima, es, generalmente, en la esfera de la informalidad en la que tienen trabajos como ambulantes, comerciantes o empleadas domésticas. Además, tienden a depender de sus lazos de parentesco y su expectativa no siempre es quedarse en la ciudad, sino ir en momentos específicos del año a trabajar.

Por otro lado, un tema que compete a la presente investigación es el rol doméstico y el rol público, o la vida familiar y la vida laboral. La literatura actual propone y discute que la división sexual del trabajo y las claras desigualdades entre hombres y mujeres en las esferas públicas y privadas se han transformado con los procesos de industrialización y, después, con la globalización, por lo que ha habido un aumento en la inserción laboral de mujeres en las últimas décadas (Alcañiz, 2015). Como explica Alcañiz, las mujeres se insertan en el trabajo remunerado con una identidad de género que está socializada y asociada a roles domésticos y de cuidado. Así, diversos autores han denominado a la duplicidad de acciones y responsabilidades que las mujeres deben de cumplir como "doble jornada", según Friedman, o "doble presencia”, de acuerdo con Balbo (Alcañiz, 2015).

El concepto de "espacio doméstico" y su discusión en la literatura sigue siendo importante para analizar y comprender mejor la realidad de la población femenina. Para Murillo, el espacio doméstico es aquel en el que se desarrollan actividades en torno a los afectos, la crianza y el cuidado, y en donde se cubren las necesidades personales de cada miembro de la familia (Luque, 2012). Este es un espacio en el que las mujeres han sido las actoras principales y articuladoras; sin embargo, esto no significa que tengan condiciones de igualdad frente a los demás miembros o que gocen de algún tipo de poder. Para Collin, las mujeres no se desarrollan como seres individuales dentro de la casa, sino que únicamente cumplen sus roles de madre o esposa y dedican siempre su tiempo a alguien más (Luque, 2012). El manejo de los horarios de las mujeres en el espacio doméstico es en función de los otros, lo que contribuye a consolidar aún más la idea que sugiere que las mujeres no tienen una autonomía propia en el espacio doméstico y su rol está en función a las necesidades de los miembros del hogar (Luque, 2012).

En la misma línea, también es relevante mencionar algunas ideas de la producción literaria en torno al cuidado. Como menciona Cárdenas (2016), el cuidado es una institución social que permite la reproducción tanto material como social de las personas. Entendido desde sus diferentes dimensiones, el cuidado puede provenir del Estado, de algunas instituciones y de la unidad familiar. Con respecto a esta última, Salazar, Jimenez y Wanderly comentan que la función de la familia de brindar cuidados a sus miembros tiende a estar repartida por roles arquetípicos de lo que el hombre y la mujer deben ser y hacer. En el caso del hombre, como esposo y padre, debe brindar sustento económico, el cual está relacionado al ámbito público y, por 
otro lado, la mujer, como madre y esposa, debe quedarse en el espacio doméstico brindando unidad y cariño (Cárdenas, 2016).

Las definiciones e ideas que plantean autores en torno al "espacio privado" y "espacio público" también son necesarias de mencionar. Con respecto al primero, este es entendido como un espacio en el que la persona puede desarrollar su individualidad y encargarse de sí misma (Murillo, 1996). Según Luque (2012), este es un espacio en el que el individuo puede disfrutar y ocuparse de sus temas personales sin tener que cumplir algún rol en particular. Sin embargo, en el caso de las mujeres, es difícil poder mantener un equilibrio con el espacio privado y doméstico, puesto que sienten la presión de las obligaciones sociales impuestos a sus roles de género (Luque, 2012). En esta línea, se plantea que el espacio público está en relación directa con el ser social; tiene que ver con el ser percibido y observado por los demás. Es donde los individuos realizan diferentes actividades e interactúan con otras personas fuera del círculo familiar (Luque, 2012). De acuerdo con esto, para Murillo, el hombre siempre ha tenido un estrecho vínculo con lo público, puesto que, a través de la historia y con la revolución industrial, el rol masculino se ubicó en las fábricas o el lugar de trabajo, fuera del ámbito doméstico y en mayor contacto con las esferas políticas, económicas y sociales (1996).

Por último, dentro de la reciente literatura que se ha producido en torno al fenómeno de la pandemia que seguimos viviendo y experimentando, algunos artículos e informes se han enfocado en la situación de las mujeres durante este contexto. La Comisión Interamericana de Mujeres (2020) publicó un informe titulado Covid-19 en la vida de las mujeres. En este se explica que, durante los meses del confinamiento entre marzo y junio del 2020 (en la mayoría de países), se ha exacerbado la "crisis de los cuidados", ya que el hogar se ha convertido en un espacio en el que "todo ocurre" y, por lo tanto, las tareas domésticas, asociadas al papel de madre y mujer, han aumentado. Asimismo, en el trabajo realizado por Burneo y Trelles (2020) en el departamento de Piura durante los primeros meses de la pandemia, se señala que "para las mujeres, el retorno ha significado un cambio muy fuerte: de tener una relativa independencia económica, la gran mayoría de ellas ha pasado a dedicarse solo a su casa y a depender enteramente de su esposo" (p. 61). De acuerdo al texto, se explica que la población femenina se encuentra en una mayor situación de vulnerabilidad durante el contexto de aislamiento social, ya que Piura es un "lugar donde las oportunidades laborales son mínimas y están dirigidas hacia los hombres" (Burneo y Trelles, 2020, p. 61), por lo que las mujeres tienen dificultades para encontrar trabajo en los espacios de retorno. En la misma línea de cuidados, la autora Pérez, explica que, en el Perú, a pesar de que en las últimas décadas la situación laboral de las mujeres ha experimentado transformaciones, la pandemia por la COVID-19 evidencia que "su posición no ha cambiado en torno a su vínculo con el hogar. Salvo poquísimas excepciones, sigue siendo la encargada de las tareas relativas al funcionamiento de la casa y de la familia" (Pérez, 2021, p. 34). De acuerdo a Osorio, durante el año 2020, los hogares se han convertido 
en "las unidades primarias de producción y reproducción básica de alimentos, mantenimiento, actividades educativas y de recreación, entre otras” (2021, p. 48).

\section{Cambios en las rutinas, acciones y responsabilidades a raíz de la pandemia y la migración}

Uno de los objetivos del trabajo de campo realizado fue identificar las acciones y tareas que realizan las mujeres en los lugares de retorno desde que regresaron de Lima. A través de las entrevistas con las tres informantes fue posible tener un acercamiento a las actividades y rutinas que realizan en sus hogares. Sin embargo, para poder comprender esto de una mejor manera, es necesario establecer una comparación con lo que hacían previamente a la pandemia y durante la cuarentena en Lima en relación al ámbito laboral, escolar y doméstico.

De acuerdo a lo que comentaron Rosa, Fiorella y María en las entrevistas realizadas, hay algunos aspectos de su vida previos a la pandemia que se diferencian de las tareas que están realizando desde que retornaron a sus hogares. El trabajo remunerado está presente en la realidad de ellas desde que son muy jóvenes. En el caso de Rosa, por ejemplo, inició a laborar como empleada doméstica desde que tenía 14 años. De igual forma, Fiorella comenzó trabajando como ayudante de cocina y mesera a la misma edad. En ese sentido, y sobre todo para Rosa, el poder ir a trabajar era parte de una rutina cotidiana a la que estaba acostumbrada desde temprana edad. Además, esto también implicaba que la organización de sus tiempos estuviera adaptada a las responsabilidades que tenía en su lugar de trabajo. Para las entrevistadas, las razones por las cuales se dedicaban a las labores domésticas en casa de terceros o trabajaban en atención al cliente respondían a la necesidad de contar con ingresos económicos para solventar sus gastos familiares y personales. Sin embargo, también se debía a las expectativas que tenían sobre lo que debía hacerse en la capital. De acuerdo a lo que compartió Rosa, a los 9 años de edad sus padres acordaron que iría a vivir a Lima junto con sus tíos paternos, ya que en la ciudad tendría una mejor educación y aprendería a hablar castellano. Durante sus primeros años en Lima, Rosa continuó en primaria y, después de algunas interrupciones, pudo terminar la secundaria. A pesar de esto, los tíos de la entrevistada la impulsaron a buscar trabajo cuando aún era una adolescente en etapa escolar debido a la necesidad de mayores aportes económicos al hogar. Desde joven, Rosa aceptó esto y empezó a comprender el trabajo y los estudios como factores relevantes en su vida; les atribuyó un valor y significado especial a las actividades remuneradas que realizaba, ya que eran parte del estilo de vida de la capital, lugar que buscó para desarrollarse en lo académico y lo laboral. De esta manera es que, hasta antes de la pandemia, la informante continuó trabajando a la par de realizar sus labores en el hogar.

Continuando con la última idea, así como Rosa, Fiorella y María también se dedicaban a las tareas domésticas antes de que iniciara la crisis sanitaria por la pandemia de la COVID-19. Ellas eran las encargadas de realizar la limpieza de la casa, la preparación 
de las comidas, cuidar a los niños pequeños, entre otras responsabilidades. Este tipo de actividades no significan algo nuevo en sus ocupaciones diarias ya que, de acuerdo a lo que comentaron en las entrevistas, son tareas con las que han estado familiarizadas desde jóvenes y que entienden como parte de su rutina diaria. En el caso de Rosa y María, por ejemplo, cuidar de la casa, de sus esposos y de sus hijos eran acciones que consideraban normales y que eran parte de otras responsabilidades como ir a trabajar o estar al tanto de los animales en la chacra. Durante las entrevistas, las informantes comentaban sobre las tareas domésticas que realizaban como parte de su estilo de vida y no parecían sentir algún tipo de fastidio por realizar estas actividades. Para Fiorella, sin embargo, quien todavía era una adolescente en etapa escolar el año pasado, este tipo de tareas representaron un incremento en las responsabilidades que tenía, un mayor sentimiento de estrés, y poco tiempo para ella misma y su recreación: “...yo me dedicaba a los quehaceres de la casa, no nos turnábamos como acá; yo sola me encargaba de todo esto y de cuidar a mis hermanos". Fiorella, quien es aun menor de edad, comentó sobre su experiencia con una clara frustración que evidenciaba la carga emocional y el exceso de responsabilidades que significó aquel periodo en el que tuvo que encargarse ella sola de las tareas domésticas en su hogar. El relato que comparte la informante permite comprender que no hay una completa aceptación del rol doméstico por parte de las mujeres, sino que es posible que existan situaciones de alto estrés generados por estas responsabilidades, así como el deseo de tener un espacio y tiempo propios.

A inicios de este año, cuando se decretaron las medidas de confinamiento como consecuencia de la COVID-19, la situación y expectativas de las informantes sufrieron transformaciones importantes $\mathrm{y}$, junto a esto, sus acciones y tareas también. Durante los meses en los que aún no retornaban a sus lugares de origen y se encontraban en cuarentena junto con sus familias, las entrevistadas comentan que tuvieron que realizar las tareas del hogar, pero que no sintieron mayores niveles de estrés con respecto a esto. De acuerdo a ellas, este fue un periodo en el que pudieron compartir las responsabilidades con sus esposos y, al mismo tiempo, enseñarles cómo se hacían algunas labores domésticas como cocinar, lavar ropa, etc. Algunas de ellas interpretaron los meses de cuarentena como un tiempo en el que crearon nuevos vínculos con sus parejas y, de alguna forma, hubo cooperación entre ellos con respecto a las tareas del hogar. Sin embargo, sí resaltan que durante estos meses estuvieron desanimadas debido al encierro y las pocas actividades que podían realizar. Para María, quien pasó la cuarentena en la casa de su cuñado en la ciudad de Lima, tales meses de reclusión tuvieron efectos negativos en su estado de ánimo, ya que únicamente se encontraba con su hija y no tenía mayores actividades que realizar durante la tarde y la noche. Es importante resaltar, también, el caso de Rosa, quien durante este periodo perdió su empleo como trabajadora doméstica. Ella y su empleadora pensaron que las medidas restrictivas no serían tan extensas; sin embargo, en el mes de abril, se le comunicó a Rosa que no podrían seguir pagándole el sueldo, por lo que perdió su empleo en una casa en la que había laborado durante varios años. Además, incluso después de haber retornado, el esposo de Rosa fue el 
único que pudo continuar con sus actividades laborales, ya que en Andahuaylas, de acuerdo a lo que comentó la informante, existe una mayor oferta de trabajo para la población masculina. Lamentablemente, a pesar de que la entrevistada disfrutara de trabajar, el aislamiento y las consecuencias de este, le impiden, hasta la actualidad, continuar con su trayectoria laboral. Por otro lado, en los meses en los que aún se encontraban en Lima y las medidas del confinamiento persistían, las entrevistadas estaban en constante comunicación con sus familiares. Según lo que comentan, algunas propuestas de retornar surgieron por parte de sus suegros o padres, ya que consideraban que sería mejor regresar a su lugar de origen, en donde no les faltaría nada de comer o un techo si es que algo más extremo sucedía.

En el caso de Fiorella, por decisión de su mamá, retornaron a Lunahuaná durante las primeras semanas del establecimiento del aislamiento social obligatorio. Regresaron a la casa de sus abuelos, donde vivían también otros familiares como sus tíos y primos. En el 2020, Fiorella comenzaría a estudiar en una preparatoria para entrar a la universidad; sin embargo, debido a que ella y sus padres consideraron que la educación virtual no sería la más adecuada, pospusieron este plan. La informante comentaba acerca de su gran ilusión por poder estudiar una carrera universitaria y, así, tener mayores oportunidades. Lamentablemente, además de la decisión de sus padres, Fiorella no pudo adaptarse a las clases virtuales, por lo que definitivamente sus objetivos trazados para el 2020 sufrieron grandes transformaciones. En cambio, durante gran parte del tiempo en Lunahuaná, Fiorella trabajó en el taller familiar, dedicándose a extraer la pulpa de las frutas que cosechaban sus abuelos y convirtiéndola en productos como mermeladas, yogures, néctares, etc. También, se encargó de realizar el proceso de etiquetado de los envases.

Con respecto a las labores domésticas en el lugar de retorno, Fiorella comenta que, en su caso, las tareas se repartían entre todas las mujeres de la casa: "Nos podíamos rotar entre todas para que no se vuelva un trabajo tedioso". Algo importante de mencionar es que, en realidad, Fiorella fue la única que retornó a un espacio en el que miembros de su familia extensa también vivían o se habían reinsertado. En el caso de Rosa y María, ellas regresaron a sus lugares de origen, pero únicamente a hogares ocupados por ellas, sus hijos y esposos. Ambas, además, se dedicaron a hacer las labores domésticas y a encargarse de las compras de los alimentos, así como también cuidar a los animales que tenían, recoger hierbas y estar al tanto de sus hijos y jugar con ellos. De acuerdo a lo que explicaron en las entrevistas, se puede entender que estas actividades en el lugar de retorno son percibidas por ellas como una continuidad de las tareas que han tenido previamente. Sin embargo, en el caso de Rosa, por ejemplo, ella tuvo que dejar de trabajar como empleada doméstica, por lo que sus actividades en el lugar de retorno fueron únicamente centradas en las actividades dentro de su hogar. Por último, otras de las actividades a las que se dedicaron y señalan como un efecto positivo dentro de todo lo negativo de la pandemia fue la de compartir con sus familiares. Para Fiorella y Rosa, por ejemplo, el retorno significó volver a pasar tiempo con sus padres, hermanos y tíos a los que 
no veían hace años o no tenían la oportunidad de visitar de manera tan seguida. Esto, además, fue entendido como una manera de sobrellevar los efectos de la cuarentena en sus estados de ánimo y los momentos de soledad que pudieron experimentar durante el aislamiento.

\section{Figura 1}

Mapa de trayectos de las tres informantes.

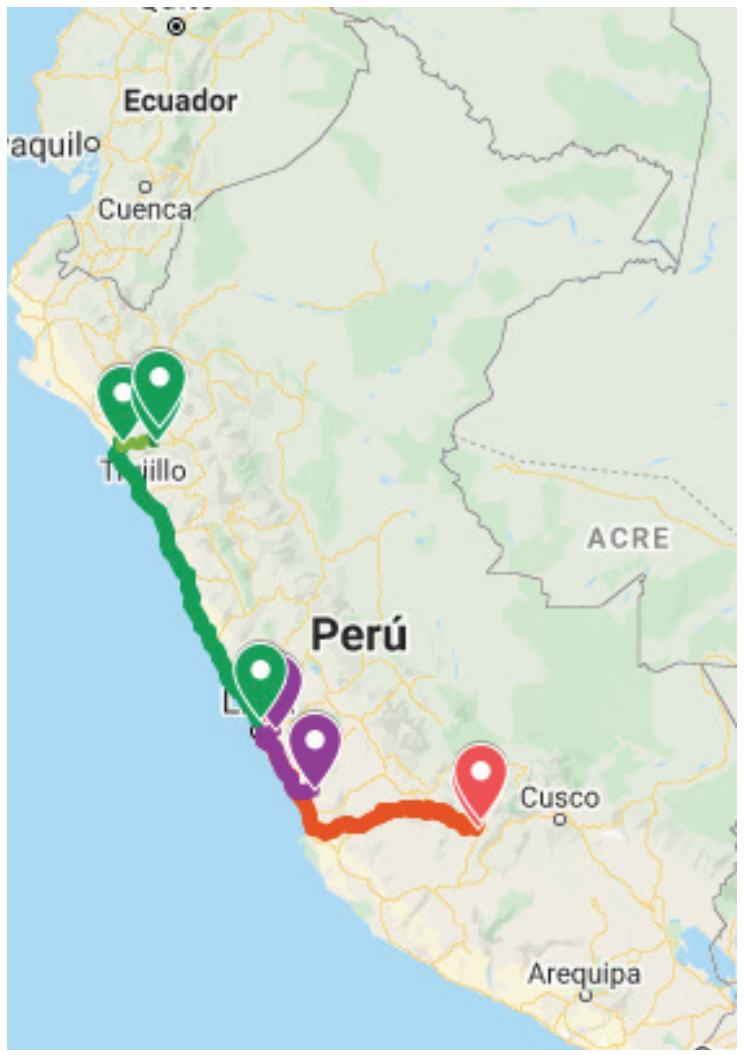

Nota: De arriba a abajo: la ruta de María, la ruta de Fiorella y la ruta de Rosa. Elaboración propia.

\section{La organización del tiempo y el descanso de las actividades}

En las entrevistas realizadas a las tres informantes, también se mencionó el tema del manejo del tiempo libre y los descansos que tienen durante el día. Esto surgió de la posibilidad de que, con las nuevas tareas adoptadas en el lugar de retorno, sea más difícil encontrar momentos del día en los que se pueda descansar. Previamente a la pandemia, tanto María como Rosa tenían organizados sus tiempos. En el caso de la última, por ejemplo, su cronograma diario se ajustaba a las necesidades que tenía y las actividades que debía realizar. Generalmente, los fines de semana servían para descansar y pasarla en familia. Sin embargo, Fiorella tuvo que enfrentarse a una sobrecarga de labores domésticas y cuidado de sus hermanos menores, las cuales 
no le permitían tener un tiempo para descansar o realizar actividades recreativas. Durante la cuarentena, las dos entrevistadas que se quedaron algunos meses en Lima antes de decidir retornar comentan que sus mañanas eran atareadas y que cuando se reactivaron algunas actividades económicas debían levantarse más temprano, ya que tenían que hacer el desayuno para sus esposos antes de ir a trabajar. Lo que explica María, por ejemplo, es que, ligado al estrés del encierro, se sumó, para ella, el aburrimiento de no saber qué hacer durante las tardes, ya que se encontraba en una vivienda que no era suya y en la que no tenía más conocidos con quienes conversar en los momentos de descanso.

En los lugares de retorno, las entrevistadas explican que no necesariamente sienten que ha aumentado su carga doméstica, pero que es posible que la organización de sus tiempos haya variado. Tanto María como Rosa son madres de familia, por lo que sus actividades diarias deben ser compartidas con sus hijos. Lo que comentan las entrevistadas es que, a pesar de que se dedican a las tareas domésticas y, en el caso de Fiorella, también a trabajar, no sienten que todo su horario sea consumido haciendo este tipo de actividades. Para María y Rosa, quienes solo viven con sus hijos y esposos, las labores domésticas son realizadas durante la mañana y en la tarde cuentan con más tiempo para apoyar a los menores y descansar. En el caso de Fiorella, por otro lado, a pesar de que trabajó en el taller de su familia durante el día y la tarde, comenta que su horario estaba organizado de tal manera que pudo disfrutar de horas libres durante el día para realizar actividades que a ella le agradaban.

En general, lo que comentan las informantes es que, aun en sus lugares de retorno, sí tienen espacio en el día para realizar actividades que disfrutan hacer. María, por ejemplo, disfruta de tejer durante sus tardes libres. Además, en algunas ocasiones suele realizar esta actividad en compañía de sus sobrinas, quienes viven cerca. Para Fiorella, regresar a Lunahuaná significó poder reorganizar de una mejor manera su horario y descansar de toda la carga de trabajo que tenía en su hogar previamente a la pandemia. La informante comenta que distribuyó el tiempo de tal manera que, además de apoyar en el taller y en las labores domésticas, también podía realizar diariamente una de sus actividades favoritas: leer.

En la línea de todo lo descrito, uno de los objetivos de la presente investigación era poder identificar las tensiones que las mujeres percibían sobre sus roles en el lugar de retorno. A pesar de que una de las ideas previas que se tenía en relación a esto era que las informantes no dispondrían de tiempo libre para descansar y relajarse, lo que comentan las entrevistadas es que no consideran que esto sea un problema. Las informantes expresaron que no sienten que se enfrentan a conflictos mayores o algunas situaciones complejas. Para Rosa y María, por ejemplo, el haber regresado a sus hogares, Andahuaylas y Llapa, respectivamente, ha sido algo positivo en su plan de vida y están satisfechas con las actividades que realizan. Sin embargo, también es necesario tomar en cuenta que algunas de las tareas que se han agregado a sus quehaceres domésticos, debido a la pandemia, son tediosas. Este es 
el caso del cuidado de los hijos, tema que, aunque no fue señalado explícitamente como una carga para ellas, sí representa un cambio a lo que generalmente estaban acostumbradas las informantes.

\section{Las mujeres y el cuidado durante la pandemia}

Las entrevistadas son madres, y hermanas de niños y niñas menores de 8 años. En el caso de Rosa, tiene un hijo llamado Diego que se encuentra en segundo grado de primaria. Fiorella, por otro lado, tiene una hermana de 7 años y un hermano de 3 años; mientras que María tiene una hija que está iniciando la primaria y tiene 6 años de edad. Previamente a la pandemia, la organización del tiempo de las mujeres y las actividades que realizan durante el día se alineaban con las responsabilidades que tenían con los niños. Fiorella, por ejemplo, pasó su verano a cargo de sus hermanos menores. Según lo que cuenta la entrevistada, pasaba todo el día junto a ellos, realizando tareas como bañarlos, cambiarlos, cocinar sus comidas y también jugar con ellos. Para ella, estos fueron meses agotadores en los que no tenía el tiempo necesario para poder realizar actividades que ella quería hacer. Asimismo, Rosa comenta que, en su cronograma de un día cotidiano previo a la pandemia, despertaba a su hijo, lo bañaba, desayunaban juntos y lo llevaba al colegio para luego dirigirse a su trabajo. Después de esto, una conocida se encargaba de recogerlo del colegio y estar con él en casa hasta que Rosa llegaba después de su jornada laboral y preparaba la cena para todos.

De acuerdo a lo que comentan las entrevistadas, desde que comenzó la pandemia y el Estado decretó que las clases serían virtuales, se les ha agregado una nueva responsabilidad a sus tareas domésticas y de cuidado: enseñarles a los niños. En el caso de Fiorella, según lo que comenta, ella no cree que su hermana necesite que la apoye constantemente con las clases, pero sí reconoce que debe estar pendiente a que esté prestando atención. Por otro lado, María y Rosa sí explican que encargarse de los estudios de sus hijos, ayudarlos en sus tareas y, sobre todo, estar presentes mientras los menores escuchan clases son nuevas responsabilidades que han surgido como efecto de la coyuntura de la COVID-19. Estas actividades, de las que ahora también tienen que hacerse cargo las informantes, generan un impacto en la manera en la que organizan su día. Según lo que comentaron, tratan de realizar la mayoría de labores durante la mañana, tales como cocinar, limpiar, cuidar a los animales, entre otras cosas, para poder dedicar las tardes a acompañar a sus hijos y guiarlos en sus clases. Durante las entrevistas realizadas, las informantes comentaron que preferían que estas fueran después de las 5 o 6 de la tarde, ya que este era el momento en el que tenían un tiempo para descansar después de haber terminado las tareas con sus hijos. Asimismo, Rosa y María perciben algunas dificultades en estas nuevas responsabilidades. Según lo que explicó esta última, a veces es complicado poder comprender los temas que le enseñan a su hija: "Muy complicado, a veces no le entendíamos, no sabía cómo para hacerlo". 
Por otro lado, el retorno a los lugares de origen de las entrevistadas y el aislamiento social obligatorio, suscitaron que ellas sean las personas más cercanas con las que sus hijos o hermanos tengan contacto durante el día. Esto se debe a que sus esposos -y, en el caso de Fiorella, su mamá- comenzaron a trabajar unos meses después de establecida la cuarentena. Entonces, las principales personas con las que los niños pueden interactuar, conversar y jugar son sus madres y hermanas. Según lo que comenta Rosa, por ejemplo, trata de salir a pasear con Diego y aprovechar que se encuentran en un lugar más amplio que la casa que tenían en Lima. Asimismo, María explica que, durante estos meses, le ha enseñado a jugar voley a su hija, actividad que ambas disfrutan hacer junto con las sobrinas de la entrevistada. En general, como ahora pasan todo el día con los niños, las informantes tienen que estar constantemente pendientes de qué actividades están realizando los menores, cuidando que no estén en peligro, o jugando con ellos.

\section{Reflexión a modo de cierre}

Como se ha tratado de describir en las páginas anteriores, el proceso de retorno de las mujeres migrantes ha generado ciertos cambios en su organización y rutina cotidiana. El presente trabajo es un acercamiento a las experiencias que han tenido las mujeres retornantes con respecto a sus nuevos roles en el lugar al que retornaron. A partir de la información que se pudo obtener de las entrevistas realizadas a Fiorella, Rosa y María es posible plantear que hay algunos roles de las mujeres que han cambiado, otros que se han mantenido similares y que, también, algunos nuevos han aparecido. A pesar de que el objetivo del presente trabajo sea más descriptivo que analítico, es posible identificar la relación entre la información obtenida y los conceptos teóricos previamente explicados. Es evidente que el espacio doméstico ha tomado una mayor importancia en los meses de la cuarentena, ya que durante una cierta cantidad de meses todos los miembros de la familia estaban presentes e interactuaban en todo momento. Asimismo, el papel de las madres y hermanas ha pasado a ser más relevante, a pesar de que es posible que otros integrantes, como los esposos, hayan empezado a ser sujetos activos en las actividades domésticas. Por otro lado, como otros estudios recientes señalan, el cuidado se ha vuelto central en esta época y mayormente recae en las mujeres, ya que se asume socialmente que ellas deben velar por la comodidad de las demás personas en el círculo familiar. Con respecto al espacio privado, de acuerdo a lo que comentan las informantes, previo a la pandemia y al retorno, las actividades que realizaban para disfrute propio y descanso no eran tan evidentes; sin embargo, durante el tiempo en el que han estado en su lugar de origen, el espacio privado sigue siendo difícil de delimitar, sobre todo con la necesidad de estar pendientes de la seguridad y recreación de los menores en sus hogares. Además, la pandemia de la COVID-19 también las ha llevado a cambiar algunos planes de vida que tenían pensado ejecutar durante este año o en el futuro, por lo que es necesario analizar qué otras transformaciones en las rutinas se seguirán generando. En general, es necesario continuar investigando sobre cómo las mujeres están viviendo y sintiendo esta época, qué dificultades han experimentado y cuáles son las expectativas que tienen a futuro. 


\section{Referencias bibliográficas}

Alcañiz, M. (2015). Género con clase: la conciliación desigual de la vida laboral y familiar. Revista Española de Sociología, (23), 29-55. http://repositori.uji.es/xmlui/bitstream/ handle/10234/150765/66900.pdf?sequence=1\&isAllowed $=y$

Burneo, M y Trelles, A. (2020). Migración de retorno en el Alto Piura en el contexto de pandemia por la COVID-19. Centro de investigación y promoción del campesinado. http:// www.cipca.pe/sites/default/files/documents/files/Investigaci $\% \mathrm{C} 3 \% \mathrm{~B} 3 \mathrm{n} \% 20$ Retornantes.pdf

Comisión Interamericana de Mujeres. (2020). Covid-19 en la vida de las mujeres: Razones para reconocer los impactos diferenciados. Organización de los Estados Americanos. https:// www.oas.org/es/cim/docs/ArgumentarioCOVID19-ES.pdf

Luque, M. (2012). De lo privado y doméstico a lo público transformaciones de las relaciones de género en las mujeres de los sectores populares de Lima Metropolitana [Tesis de pregrado, Universidad Nacional Mayor de San Marcos]. http:/cybertesis.unmsm.edu.pe/bitstream/ handle/20.500.12672/299/Luque_vm.pdf?sequence=1\&isAllowed=y

Murillo, S. (1996). El mito de la vida privada. De la entrega al tiempo libre. Siglo XXI de España.

Osorio, S. (2021). Encuentros y desencuentros entre los cuidados y la economía. En Oxfam (Ed.), Tiempos de cuidados (pp.19-43). Oxfam. https://oi-files-cng-prod.s3.amazonaws.com/ peru.oxfam.org/s3fs-public/file_attachments/Tiempos-de-Cuidados-Peru.pdf

Paerregaard, K. (2007). La migración femenina: estrategias de sostenimiento y movilidad social entre peruanos en España y Argentina. Anthropologica, 25(25), 61-82. http://www. scielo.org.pe/scielo.php?script=sci_arttext\&pid=S0254-92122007000100003

Pérez, L. (2021). Los cuidados en los tiempos de coronavirus y más allá: Mujeres peruanas, trabajo doméstico, dilemas, y soluciones. En Oxfam (Ed.), Tiempos de cuidados (pp. 4371). Oxfam. https://oi-files-cng-prod.s3.amazonaws.com/peru.oxfam.org/s3fs-public/file attachments/Tiempos-de-Cuidados-Peru.pdf 